Fingergelenke

\section{Was ist die beste Strategie für die Behandlung der MCP-Prothesenluxation?}

Wanderman $\mathrm{N}$ et al. Outcomes Following Acute Metacarpophalangeal Joint Arthroplasty Dislocation: An Analysis of 37 Cases. J Hand Surg Am 2018; 43: 289.e1 - 289.e6

Über die Ergebnisse einer Behandlung luxierter Metakarpophalangealgelenk-Arthroplastiken gibt es wenige Informationen. Handchirurgen der Mayo-Klinik in Rochester, USA, bewerteten die chirurgischen und nicht operativen Behandlungsmodalitäten luxierter Grundgelenkprothesen.

Für die retrospektive Analyse wurden aus insgesamt 816 MCP-Gelenkarthroplastiken, operiert von 9 Chirurgen über einen Zeitraum von 14 Jahren, 37 (4\%) akute MCP-Gelenkluxationen von 26 Patientinnen und einem Patienten ausgewählt, die eine Intervention durch einen Arzt erforderten. Bestimmt wurden aus den verfügbaren Unterlagen Behandlungsmodalitäten, Komplikationen und daraus folgende Operationen. Die Luxationen mussten radiologisch nachweisbar sein. Bei den Implantaten handelte es sich um 28 non-constrained Implants (17 Pyrocarbon- und 11 Oberflächenersatz-Prothesen) und 9 Silikonimplantate. Die Auswertung umfasste die Behandlung von Luxationen nach primärer $(n=30)$ und revidierter $(n=7)$ Grundgelenkarthroplastik.

Die Zeit zwischen Implantation und Auftreten der Luxation betrug median 1,3 Jahre. Die den Luxationen zugrunde liegenden Ursachen waren in 6 Fällen eine Implantatfraktur, in 2 Fällen eine Komponentenlockerung und in 29 Fällen eine Insuffizienz der Weichteile. Implantatfrakturen traten nur bei Silikonimplantaten auf. Von den akuten Luxationen wurden 14 initial nicht chirurgisch durch geschlossene Reposition und Orthesenherstellung behandelt. Diese Verfahren schlugen alle letztendlich fehl. Einschließlich einiger der fehlgeschlagenen früheren Versuche wurden 18 Weichteil- stabilisierungsverfahren und 21 Revisionsarthroplastiken durchgeführt. Letztere erfolgten isoliert oder in Kombination mit Weichteilstabilisierungsverfahren.

Ein Eingriff war erfolgreich, wenn nach mindestens 6 Monaten Nachbeobachtungszeit keine klinischen und radiologischen Anzeichen einer Instabilität nachweisbar waren. Danach hatten alleinige Weichteilstabilisierungsverfahren eine Erfolgsrate von $28 \%$, um ein stabiles MCP-Gelenk zu erreichen und die Revisionsendoprothetik eine Erfolgsrate von $71 \%$. Die Subgruppenanalyse ergab eine Erfolgsquote von $86 \%$ für die Revision der Silikonimplantate und ein 5-JahresÜberleben ohne Instabilität von $80 \%$. Bei den nicht gekoppelten Revisionsarthroplastiken, darunter fielen 4 Pyrocarbonund 3 Oberflächenersatz-Arthroplastiken, betrug die Erfolgsrate $43 \%$ mit einem 5 -Jahres-Überleben von $36 \%$ ohne Instabilität.

\section{FAZIT}

Die besten Ergebnisse für eine akute MCP-Gelenkendoprothesen-Luxation werden für Silikonimplantate durch eine Revisionsarthroplastik erreicht, schlussfolgern die Autoren.

Richard Kessing, Zeiskam 